\title{
LAS DIFERENCIAS SALARIALES EN MÉXICO Y SU MEDICIÓN
}

\author{
Adalberto García Rocha * \\ El Colegio de México
}

\section{INTRODUCCIÓN}

Este tRABajo constituye la parte nacional de un proyecto de comparación de salarios que, junto con otros dos estudios (uno de consumo y otro de precios), es llevado a cabo a nivel latinoamericano bajo lo coordinación de Brookings Institution. El estudio global funciona bajo la denominación de ECIEL (Estudios Conjuntos de Integración Económica Latinoamericana) y, en el caso del estudio de diferencias salariales, cada instituto participante contribuye con un estudio nacional y con información y colaboración técnica para una comparación internacional.

Aquí se maneja el problema de medir las diferencias salariales considerado como un problema de concentración similar al que aparece en [1]. Se hace luego una aplicación del método de medición al caso de una muestra de empresas de la ciudad de México. Cabe aclarar que el enfoque presente no considera la característica sin duda más interesante del fenómeno salarial, a saber, su evolución en el tiempo; sin embargo, con información suficiente, el problema puede ser manejado con el método que se expone en términos estático-comparativos.

\section{CONSIDERACIONES GENERALES SOBRE EL FENÓMENo SALARIAL}

La determinación de la estructura y el nivel de los salarios de una economía es un problema viejo en la teoría económica [2] [3]; los diversos enfoques, desde el concepto del fondo de salarios, hasta el enfoque distributivo del modelo neoclásico, examinan el problema de la distribución de los pagos a los factores y las causas de las fluctuaciones de éstos. El salario es uno de los vehículos más importantes de distribución del ingreso así como uno de los elementos más socorridos de política económica, por lo cual resulta interesante analizar los sentidos de sus disparidades con el objeto de descubrir sus posibles determinantes. A este fenómeno se encuentra directamente vinculado

* El autor agradece a Pedro Uribe sus valiosas sugerencias en el sentido de utilizar la medida de información que se explica más adelante para la medición de las diferencias. Igualmente agradece importantes comentarios de Raúl de la Peña (CEED) y Daniel Murayama (CEED), en relación con varios aspectos del trabajo. Se agradece también a los centros de cálculo de la UNAM y Petróleos Mexicanos el haber facilitado tiempo en sus respectivas computadoras para el proceso que se trata en la parte final del trabajo. 
el de la movilidad de la fuerza de trabajo, en la medida en que el salario constituye un estímulo al cambio de empleo.

De acuerdo con la teoría de la distribución y la producción, las condiciones de operación y la estructura tecnológica de las empresas son los factores fundamentales de distribución de los pagos a los factores. El enfoque más conocido al problema es el de la teoría de la producción que define, para cada empresa, la existencia de una función que refleja cómo cada factor contribuye a la formación del producto y asimismo la magnitud de la contribución de cada uno de ellos. En dicha función, la tecnología se refleja en varias características: la facilidad para substituir los factores, el nivel de economías de escala, y otros elementos autónomos incluidos con la idea de dar cabida a la organización, la educación y cambio cualitativos difíciles de considerar explícitamente en dicha función.

Este enfoque lleva directamente a la determinación de los salarios una vez que se conocen, para cada empresa, las contribuciones que hacen cada uno de sus empleados a la formación del producto. En otras palabras, el conocimiento de funciones de producción suficientemente desglosadas permitiría derivar los niveles individuales de salarios y de aquí su estructura.

El problema de medición de las diferencias salariales resulta entonces un subproducto del problema más general de la aplicación de la teoría de la producción y la distribución a una economía. Además de varios inconvenientes teóricos involucrados, el enfoque anterior se enfrentaría al obstáculo de la falta de información para llevar a cabo un análisis de esta naturaleza. Por otra parte, existe un factor adicional en la determinación de los salarios que hace que el problema adquiera mayor complicación. Este factor adicional de gran relevancia aparente es la existencia de uniones laborales cuyo efecto sobre los salarios y su estructura conjuntamente con los factores puramente económicos, es un problema aún no dilucidado [4], [5].

De estas consideraciones surge un problema fundamental: el de establecer el grado relativo en que los factores económicos y sindicales determinan los salarios y su estructura. El problema presenta, además, sus rasgos más interesantes desde el punto de vista dinámico, ya que la influencia relativa de ambos elementos ha cambiado en el tiempo y seguramente recibe influencias extra-económicas que inciden sobre el fenómeno salarial. Todo esto sin considerar la influencia de otros factores que juegan un papel importante, por ejemplo: la política fiscal y la de salarios mínimos.

De todo lo anterior se puede concluir que una explicación satisfactoria de los fenómenos salariales no puede desarrollarse sin caer en el contexto de un problema económico mucho más amplio.

Otro enfoque consiste en tratar de simular el proceso de determinación de los salarios en función de las consideraciones que hace el empresario o el contratante al ofrecer la remuneración a la persona que solicita empleo, y también respecto de aquellos elementos que se toman en cuenta para modificar el salario. En dicha simulación se acude a variables en las que en realidad se intenta reflejar la productividad del empleado. De este modo, dentro de los estándares de salarios corrientes, el individuo contratante se atiene a características 
tales como la educación, la responsabilidad, la experiencia, la iniciativa, la antigüedad, los riesgos, etc., implicados en un cargo, y que constituyen para el empleador los principales elementos de cuantificación del sueldo. Debe hacerse notar que este enfoque se hace en términos de las características del puesto, que deben ser satisfechas por el individuo contratado, y el fenómeno global puede tratarse desde el punto de vista del grado de adaptación de la fuerza de trabajo a los empleos existentes, según los requisitos que deben ser satisfechos para desempeñarlos. Es decir, que finalmente este fenómeno de adaptación viene a determinar el nivel y la estructura de los salarios. Originalmente, el análisis propuesto para los estudios nacionales de salarios del programa ECIEL fue de este tipo, pero por falta de información en el caso de México, el trabajo se reduce al problema de medir las diferencias solamente.

\section{EL SENTIDO Y LA MEDICIÓN DE LAS DIFERENCIAS}

Las diferencias salariales pueden considerarse de acuerdo con dos aspectos: las disparidades ocupacionales debidas a los distintos niveles de calificación de los asalariados (escala salarial), y las diferencias "geográficas" que se observan en una misma categoría ocupacional (empleo o grupo de empleos) en las distintas unidades económicas (empresas, grupos, sectores, etc.).

Las diferencias del primer tipo reflejan en cierta medida la contribución diferencial que hacen los empleados a la formación del producto de la empresa que los tiene contratados. En este sentido, la escala de las remuneraciones se establece supuestamente según el grado de calificación del empleado. De este modo, dentro de cada empresa, los salarios van desde los niveles inferiores de los obreros manuales de baja calificación, hasta los niveles superiores de los técnicos, profesionistas y personal administrativo de alta jerarquía. En este sentido, las diferencias pueden verse afectadas por la escasez de ciertos tipos de personal, y por este medio las diferencias reciben el efecto directo del mercado de estos empleos.

Por su parte, las diferencias geográficas son básicamente el resultado de diferencias en los niveles de carestía o bien, cuando éstas se aíslan, de la importancia relativa que tienen los diferentes cargos en las distintas empresas. Por ejemplo, un químico no tiene la misma importancia para la industria química que para la industria alimenticia. En el fondo, este fenómeno tiene que ver con las diferencias tecnológicas que hacen que la productividad de un cargo de igual denominación difiera de una empresa a otra.

Estos sentidos de importancia económica que puedan tener las diferencias salariales discutidas en los párrafos anteriores sirven de guía para cuantificarlas.

Si se cuenta con información sobre retribuciones y mano de obra según una clasificación por empleos y empresas, a saber,

$S_{i j}=$ retribuciones al empleo $i$ hechas por la empresa $j$ y

$$
Q_{i j}=\text { mano de obra } i, j
$$


que podrían provenir de un censo o una muestra, la nómina total sería

$$
S=\sum_{i j} S_{i j}
$$

y la mano de obra total

$$
Q=\sum_{i j} Q_{i j}
$$

de lo cual, el salario medio global resulta ser $S / Q$.

La información sobre retribuciones y mano de obra, según las definiciones anteriores, permite aproximar las distribuciones discretas de probabilidad

y

$$
\left\{w_{i j}\right\}=\left\{\frac{S_{i j}}{S}\right\}
$$

$$
\left\{l_{i j}\right\}=\left\{\frac{Q_{j i}}{Q}\right\}
$$

E1 término $w_{i j}$, representa la probabilidad de encontrar una unidad retribuida al empleo $i$ por la empresa $j$, y $l_{i j}$ representa la situación correspondiente a la mano de obra. Debe notarse que

y que

$$
Q \leqslant w_{i j}, l_{i j} \leqslant 1
$$

$$
\sum_{i j} w_{i j}=\sum_{i j} l_{i j}=1
$$

Considérese ahora una situación en la que $w_{i j}=l_{i j}$, para todo $i \mathrm{y}$ todo $j$, es decir, para todas las empresas y todos los empleos. En este caso, el significado económico es que en todos los empleos y en todas las empresas los salarios son idénticos, y asimismo, idénticos al salario medio global.

La situación real no es, desde luego, la de salarios iguales, pero este caso extremo puede servir de patrón de comparación de las disparidades. En otras palabras, la medida en que $\left\{w_{i j}\right\}$ difiera de $\left\{l_{i j}\right\}$ puede tomarse como la magnitud de las diferencias salariales. Estas pueden cuantificarse de muchas maneras, pero en general cualquier índice o medida de ellas debe cumplir con los siguientes criterios:

a) El índice debe ser cero cuando $\left\{w_{i j}\right\}=\left\{l_{i j}\right\}$. Este es el caso extremo en que las disparidades son nulas.

b) Debe crecer cuando la diferencia entre $\left\{w_{i j}\right\}$ y $\left\{l_{i j}\right\}$ crece. Conviene aclarar que la disimilitud entre ambas distribuciones no implica necesariamente una relación de orden, es decir, la idea no es la de establecer una definición que nos permita decir, por ejemplo, que $\left\{w_{i j}\right\} \leqslant$ $\left\{l_{i j}\right\}$. De aquí surge una tercera condición.

c) Que la medida sea positiva siempre que $\left\{w_{i j}\right\}$ y $\left\{t_{i j}\right\}$ no sean iguales. 
Para aclarar un poco el concepto, supóngase que la proporción de jefes de personal en la empresa $x$ es inferior a la proporción de salarios correspondientes; esto significa que en algún otro empleo $\mathrm{y} / \mathrm{o}$ en alguna otra empresa debe presentarse el caso contrario. Desde el punto de vista de los criterios enunciados, la medida que se utilice dará magnitudes idénticas si la situación se invierte, es decir, si la proporción de jefes de personal en la empresa $x$ es superior a los salarios correspondientes.

No obstante, si el índice es susceptible de ser descompuesto en forma aditiva, se puede averiguar cuál de los dos casos está presente.

La medición de las diferencias de salarios se hará mediante la expresión :

$$
I=\sum_{\imath j} w_{i j} \log \frac{w_{i j}}{l_{i j}}
$$

que en teoría de la información se conoce con el nombre de información indirecta con probabilidad a priori $l_{i j}$ y probabilidad a posterio. ri $w_{i j}[6]$.

Si $w_{i j}=l_{i j}$; para todo $i$ y todo $j$, la expresión (1) se convierte en

$$
\sum_{i j} w_{i j} \log \frac{w_{i j}}{l_{i j}}=\sum_{i j} w_{i j} \log 1=\sum_{i j} w_{i j} \cdot 0=0
$$

lo cual cumple con el criterio a) expuesto.

Por otra parte, es posible demostrar que la desigualdad

$$
\sum_{i j} w_{i j} \log \frac{w_{i j}}{l_{i j}}=\frac{1}{2} \sum_{i j} w_{i j}\left(w_{i j}-l_{i j}\right)^{2}
$$

es cierta [7]. En palabras, $I$ es siempre mayor que una cantidad siempre positiva. Con esto se satisfacen los requisitos $b$ ) y $c$ ).

Lo que sigue es precisamente obtener la estructura de $I$ a través de una descomposición que refleje los rasgos interesantes de las diferencias, de acuerdo con lo expuesto al principio. Nótese que la descomposición es múltiple y que el criterio de seleccionar alguno, obedecerá a las diferencias de interés económico.

Antes de proceder a la descomposición, es indispensable hacer algunas definiciones adicionales, respecto a la agregación de que son susceptibles $\left\{w_{i j}\right\}$ y $\left\{l_{i j}\right\}$. Las empresas pueden agregarse en grupos para obtener ramas o sectores de actividad económica $\mathrm{y}$, por su parte, los empleos pueden agregarse para formar grupos ocupacionales. Para entender mejor esto, véase el cuadro de la página siguiente.

De acuerdo con el cuadro, se define $W_{i h}=\sum_{j \varepsilon S_{h}} w_{i j}$ como la proporción de la nómina total pagada al empleo $i$ por el sector $h$ constituido por las empresas $j \varepsilon S_{h}$, ${ }^{*}$ asimismo $W_{g j}=\sum_{i \varepsilon G_{g}} w_{i j}$ representan la proporción de salarios retribuida al grupo ocupacional $g$ por la empresa $j$.

\footnotetext{
* $j \varepsilon S_{h}$ significa las empresas $j$ que están en el sector $h$.
} 


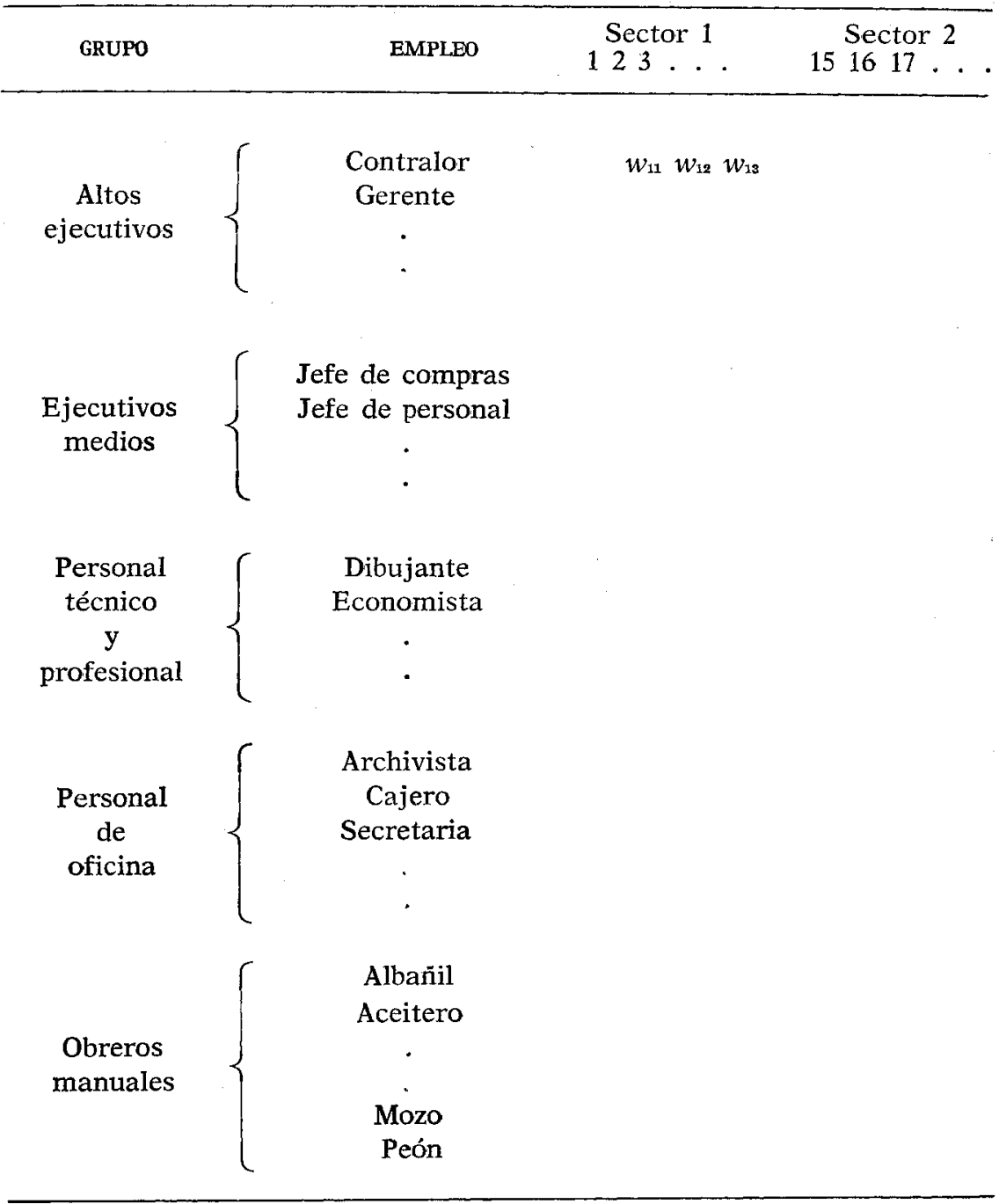


La agregación doble $W_{g h}=\sum_{i \varepsilon G_{g}} \sum_{j \varepsilon S_{h}} w_{i j}$ representa entonces la retribución al grupo ocupacional $g$ por el sector $h$.

Las dos agregaciones parciales y esta última, son enteramente similares para el caso de la distribución $l_{i j}$.

La agregación en ambos sentidos puede representarse.

\begin{tabular}{|c|c|c|c|c|c|}
\hline Grupo & 1 & 2 &..$h$. & $\ldots H$ & \\
\hline 1 & $\mathcal{W}_{11}$ & $w_{12}$ & & & $w_{1}$. \\
\hline 2 & & & & & \\
\hline$\cdot$ & & & & & \\
\hline . & & & & & \\
\hline - & & & & & \\
\hline$g$ & & & $w_{g h}$ & & \\
\hline . & & & & & \\
\hline$\cdot$ & & & & & \\
\hline$\cdot$ & & & & & \\
\hline . & & & & & \\
\hline • & & & & & \\
\hline$G$ & & & & $w_{G B}$ & $w_{a}$ \\
\hline
\end{tabular}

que reflejaría el caso en el que sólo se cuenta con información para $G$ grupos ocupacionales y $H$ sectores. Los totales horizontales y verticales de este último cuadro se denotarán por

$$
\begin{aligned}
& W_{g} .=\sum_{h} W_{g h} \\
& W_{. h}=\sum_{g} W_{g h}
\end{aligned}
$$

IV. DESCOMPOSICIÓN DE LAS DIFERENCIAS

Supóngase que únicamente se cuenta con información sobre retribuciones a los grupos ocupacionales, es decir, sólo son conocidas las distribuciones $\left\{W_{g} \cdot\right\}$ y $\left\{L_{g}.\right\}$. Las diferencias de salarios serían, por analogía con (1),

$$
\sum_{g=1}^{G} W_{g} . \log \frac{W_{g} .}{L_{g} .}
$$

que mide las diferencias entre los distintos grupos ocupacionales sin tomar en cuenta su heterogeneidad interna. Si $\left\{W_{g}.\right\}=\left\{L_{g}.\right\}$, los salarios medios de cada grupo son iguales entre sí; nótese que esto 
no implica que los salarios medios no tengan disparidades internas en los grupos.

Supóngase ahora que se conoce la información para los grupos ocupacionales en los distintos sectores económicos. En este caso, es posible averiguar la disparidad interna de un grupo debida a las diferencias entre los distintos sectores. En otras palabras, la distribución condicional $\left\{\frac{W_{g h}}{W_{g}}\right\}$ se refiere a la información interna del grupo $g$ y por lo tanto la disparidad correspondiente es

$$
\sum_{n} \frac{W_{g h}}{W_{g} .} \log \frac{W_{g h} / W_{g} .}{L_{g h} / L_{g} .}
$$

que de nuevo se obtiene por analogía con (1). Si esta última expresión se pondera según la importancia de los grupos ocupacionales en la nómina total $\left(W_{g}.\right)$, se suma sobre $g$ y el resultado se añade a (2), se obtiene

$$
\begin{aligned}
& \sum_{g} W_{g} \cdot \sum_{h} \frac{W_{g h}}{W_{g} .} \log \frac{W_{g h} / W_{g}}{L_{g h} / L_{g}}+\sum_{g} W_{g} \cdot \log \frac{W_{g} \cdot}{L_{g}}= \\
& =\sum_{g} \sum_{h} W_{g h} \log \frac{W_{g h} / W_{g}}{L_{g h} / L_{g} .}+\sum_{g} \sum_{h} W_{g h} \log \frac{W_{g} \cdot}{L_{g}}= \\
& =\sum_{g} \sum_{h} W_{g h} \log \frac{W_{g h}}{L_{g h}}
\end{aligned}
$$

Esta última expresión mide las diferencias salariales cuando se conocen las distribuciones agregadas $\left\{W_{g h}\right\}$ y $\left\{L_{g h}\right\}$. En vista del modo en que se obtuvo la expresión anterior, ésta consta de dos componentes: las diferencias entre los grupos ocupacionales y las diferencias internas de éstos al nivel de sector. En suma,

Diferencias entre grupos (expresión 2)

Diferencias intra grupos (expresión 3) ${ }^{+}$

Diferencias entre sectores y grupos (expresión 4)

Cuando se conoce la información al nivel de grupo ocupacional y sector, las diferencias totales alcanzan la magnitud expresada por (4). Sin embargo, dentro de cada grupo y/o sector (celda) existen a su vez diferencias. Es decir, si se cuenta con la información interna de cada celda, por ejemplo: que para el caso del sector económico 10 se conocieran los porcientos de sueldos y fuerza de trabajo correspondientes a los empleos que constituyen el grupo ocupacional 7, sería posible medir la heterogeneidad interna de la celda $(10,7)$ en cuanto a los empleos que la constituyen. En general, si se conoce la composición de empleos de la celda $(g, h)$, se pueden evaluar las diferencias: 


$$
\sum_{i \varepsilon G_{g}} \frac{W_{i h}}{W_{g h}} \log \frac{W_{i h} / W_{g h}}{L_{i h} / L_{g h}}
$$

que miden la disimilitud de los empleos dentro del grupo $g$ en el sector $h$. En otras palabras, la fórmula anterior mide diferencias entre empleos dentro de la celda $(g, h)$ que es el análogo de la expresión (2) a nivel de grupo ocupacional. De acuerdo con esto, parece natural definir, paralelamente a la descomposición agregada, las diferencias internas de los empleos dentro de la celda, o sea:

$$
\sum_{j \varepsilon S_{h}} \frac{W_{i j}}{W_{i \hbar}} \log \frac{W_{i j} / W_{i \hbar}}{L_{i j} / L_{i \hbar}}
$$

que refleja la disparidad interna del empleo $i$ dentro del sector $h$, esto es, entre las empresas que lo constituyen. Dentro de la celda, cada empleo pesa $\frac{W_{i h}}{W_{g h}}$ y por tal razón las diferencias intra empleo de la celda $(g, h)$ están dadas por

$$
\sum_{i \varepsilon G_{g}} \frac{W_{i h}}{W_{g h}} \sum_{j \varepsilon S_{h}} \frac{w_{i j}}{W_{i h}} \log \frac{w_{i j} / W_{i h}}{l_{i j} / L_{i \hbar}}
$$

Hecho lo anterior, al sumar (5) y (7) resulta

$$
\begin{aligned}
& \sum_{i \varepsilon G_{g}} \frac{W_{i h}}{W_{g h}} \log \frac{W_{i h} / W_{g h}}{L_{i h} / L_{g h}}+ \\
+ & \sum_{i \varepsilon G_{g}} \frac{W_{i h}}{W_{g h}} \sum_{j \varepsilon S_{h}} \frac{w_{i j}}{W_{i h}} \log \frac{w_{i j} / W_{i h}}{l_{i h} / L_{i h}}= \\
= & \sum_{i \varepsilon G_{g}} \sum_{j \varepsilon S_{h}} \frac{w_{i j}}{W_{g h}} \log \frac{w_{i j} / W_{i h}}{L_{i j} / L_{i \hbar}}+\log \frac{W_{i h} / W_{g h}}{L_{i h} / L_{g h}}= \\
= & \sum_{i \varepsilon G_{g}} \sum_{j \in S_{h}} \frac{w_{i j}}{W_{g h}} \log \frac{w_{i j} / W_{g h}}{l_{i j} / L_{g h}}
\end{aligned}
$$

Por su parte, cada celda contribuye con $W_{g h}$ a las diferencias totales, o sea que

$$
\sum_{g, h} W_{g h} \sum_{i \varepsilon G_{g}} \sum_{j \varepsilon S_{h}} \frac{w_{i j}}{W_{i g h}} \log \frac{w_{i j} / W_{g h}}{l_{i j} / L_{g h}}
$$

lo cual sumado a (4)

$$
\sum_{g h} W_{g h} \log \frac{W_{g h}}{L_{g h}}+\sum_{g, h} \sum_{i \varepsilon G_{g}} \sum_{j \varepsilon S_{h}} w_{i j} \log \frac{w_{i j} / W_{g h}}{l_{i j} / L_{g h}}=
$$




$$
=\sum_{i, j} w_{i j} \log \frac{w_{i j}}{l_{i j}}
$$

que es de nuevo la expresión (1).

En síntesis, las diferencias pueden dividirse en dos grandes grupos:

a) internas de las celdas; $b$ ) entre las celdas.

Las diferencias internas de las celdas pueden a su vez dividirse en:

a) Diferencias entre empleos;

b) Diferencias intra empleos.

Por su parte, las diferencias entre las celdas constan de:

a) Diferencias internas de los grupos ocupacionales;

b) Diferencias entre los grupos ocupacionales.

Toda esta descomposición, con las ponderaciones adecuadas, es aditiva, y esta flexibilidad es cómoda al interpretar el significado de cada elemento de las disparidades. Asimismo, la importancia relativa de cada una tiene sentido económico muy claro.

La característica común entre las disparidades internas de los empleos y las internas de los grupos ocupacionales, es que son el resultado de diferencias entre las empresas que constituyen el sector en el primer caso, y de las diferencias entre los sectores, en el segundo. Es decir, ambas diferencias son geográficas.

Por ejemplo, la disparidad interna del empleo "electricista", dentro del sector "industria química", es el resultado de la disparidad de salarios pagados por las distintas empresas que constituyen dicho sector.

Por su parte, las diferencias internas del grupo ocupacional "empleados de oficina" son consecuencia de la disparidad de los salarios pagados de ese grupo por los distintos sectores económicos.

Las diferencias de este tipo representan, al igual que los demás, la magnitud del estímulo a cambiar de empleo. Mientras más agudas sean las diferencias dentro de un sector, la mano de obra derivará más ventajas de un cambio de empleo dentro de un sector. Asimismo, las diferencias internas de los grupos ocupacionales son una medida de estímulo a cambiar de sector de actividad económica. Esto es válido en la medida en que el salario sea un factor de retención o de atracción, y de la facilidad de acceso a los empleos.

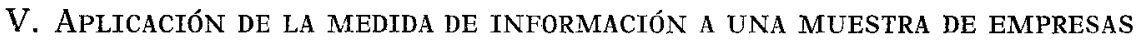
DE LA CIUDAD DE MÉxICo *

En este parte se tratará de aplicar la medida de información al caso de una muestra de empresas de la zona metropolitana de la ciudad de México [3].

La información fue recogida en diciembre de 1965 y se obtuvo de aproximadamente 150 empresas y negociaciones.

* El autor agradece al Instituto de Participación de las Utilidades en el Salario, de la Confederación Patronal de la República Mexicana, el haber facilitado generosamente la información que se manejó en este trabajo. 
Los datos que contiene se refieren a los sueldos, sueldos incentivos, prestaciones y número de personas en cada uno de 97 cargos diferentes, y que tienen la distribución siguiente:

Grupo

1. Altos ejecutivos

2. Ejecutivos medios

3. Personal técnico y profesional

4. Personal de oficina

5. Obreros manuales
Núm. de Cargos

30

La encuesta permite entonces aproximar las distribuciones $\boldsymbol{w}_{i j}$ y $l_{i j}$ requeridas para el análisis.

Una vez depurada la muestra, su tamaño se redujo a 92 empresas. La depuración consistió básicamente en eliminar a todas aquellas empresas cuya distribución de fuerza de trabajo no pareciera factible; por ejemplo: aquellas que sólo manifestaron tener un empleo.

De este modo, los arreglos $w_{i j}$ y $l_{i j}$ resultan ser de 97 empleos por 92 empresas. La agregación de los empleos es la misma de los grupos ocupacionales anteriores. Estos grupos constituyen en forma aproximada el concepto de conglomeriado de empleos * definido como una agrupación de empleos afines en cuanto al salario, la organización de la empresa, la tecnología, las políticas de ascenso, etc. Se supone entonces que las disparidades internas de estos conglomerados son más o menos bajas.

En cuanto a la agregación de las empresas, ésta se vio muy comprometida por la cantidad de información disponible. Los grupos de empresas no siguen un criterio de similaridad y por tal razón no puede garantizarse su homogeneidad interna o que correspondan al concepto de contorno salarial.** Los grupos y el número de empresas son:

I. Alimentos, bebidas, tabaco, textiles, papel y productos de papel, imprentas, editoriales e industrias conexas y productos de caucho

II. Sustancias y productos químicos, productos minerales no metálicos, exceptuando derivados del petróleo y carbón

III. Industrias metálicas básicas, productos metálicos, construcción de maquinaria, aparatos accesorios y artículos eléctricos, construcción de material de transporte e industrias manufactureras diversas 
IV. Comercio al por mayor y al por menor y seguros

V. Extracción de minerales, construcción, agua y servicios, actividades no bien especificadas, depósito y almacenaje, servicios públicos y servicios personales

En los cuadros 1 y 2 aparecen las distribuciones de los salarios $w_{g h}$ antes y después de los impuestos respectivamente. En los cuadros 3 y 4 se muestran los salarios con respecto al salario medio global.

Se aplicó a la información sobre salarios nominales la ley del impuesto vigente, y en ambos casos se hizo la medición de las diferencias.

\section{Resultados}

En los cuadros 1 y 2 aparecen las distribuciones de remuneraciones y fuerza de trabajo según la clasificación en cinco grupos ocupacionales y cinco agrupaciones de empresas ya expuesta. Puede observarse en el cuadro 1 que los dos últimos grupos ocupacionales reciben las dos terceras partes de los pagos globales, y la proporción de personas en ellos es de $82.72 \%$ del total. Esto da idea de la desproporción entre la distribución de los pagos en los tres primeros y los dos últimos grupos. En el cuadro 3 puede verse que el último grupo (trabajadores manuales) es el único que compensa la relación con el salario global, es

\section{Cuadro 1}

DISTRIBUCIÓN DE LA FUERZA DE TRABAJO Y SUS REMUNERACIONES POR GRUPO OCUPACTONAL Y RAMA DE ACTIVIDAD, 1965

Antes de los impuestos

(Porcientos)

\begin{tabular}{|c|c|c|c|c|c|c|c|}
\hline \multirow{2}{*}{ Grupo } & & \multicolumn{5}{|c|}{$S \circ c t \circ r$} & \multirow{2}{*}{ Total } \\
\hline & & $I$ & II & III & IV & $\mathbf{v}$ & \\
\hline 1 & $\begin{array}{l}\text { Renuneraci6n } \\
\text { Puerza de trabajo }\end{array}$ & $\begin{array}{l}2.14 \\
0.44\end{array}$ & $\begin{array}{l}1.84 \\
0.42\end{array}$ & $\begin{array}{l}2.62 \\
0.58\end{array}$ & $\begin{array}{l}1.95 \\
0.33\end{array}$ & $\begin{array}{l}0.95 \\
0.22\end{array}$ & $\begin{array}{l}9.50 \\
1.99\end{array}$ \\
\hline 2 & $\begin{array}{l}\text { Remuneracion } \\
\text { Fuerza de trabajo }\end{array}$ & $\begin{array}{l}6.32 \\
3.18\end{array}$ & $\begin{array}{l}4.59 \\
1.84\end{array}$ & $\begin{array}{l}3.57 \\
2.24\end{array}$ & $\begin{array}{l}3.70 \\
1.92\end{array}$ & $\begin{array}{l}1.49 \\
0.73\end{array}$ & $\begin{array}{r}19.67 \\
9.91\end{array}$ \\
\hline 3 & $\begin{array}{c}\text { Remuneracion } \\
\text { Fuerza de trabajo }\end{array}$ & $\begin{array}{l}1.94 \\
1.42\end{array}$ & $\begin{array}{l}1.52 \\
1.28\end{array}$ & $\begin{array}{l}1.64 \\
1.48\end{array}$ & $\begin{array}{l}0.88 \\
0.76\end{array}$ & $\begin{array}{l}0.52 \\
0.42\end{array}$ & $\begin{array}{l}6.50 \\
5.36\end{array}$ \\
\hline 4 & $\begin{array}{c}\text { Remuneracion } \\
\text { Paerza de trabajo }\end{array}$ & $\begin{array}{l}12.48 \\
10.15\end{array}$ & $\begin{array}{l}6.60 \\
6.53\end{array}$ & $\begin{array}{l}4.82 \\
6.86\end{array}$ & $\begin{array}{l}8.17 \\
9.68\end{array}$ & $\begin{array}{l}6.05 \\
4.19\end{array}$ & $\begin{array}{l}38.12 \\
37.41\end{array}$ \\
\hline 5 & $\begin{array}{l}\text { Remuneración } \\
\text { Fuerza de trabajo }\end{array}$ & $\begin{array}{r}8.38 \\
14.28\end{array}$ & $\begin{array}{l}5.04 \\
9.20\end{array}$ & $\begin{array}{r}9.52 \\
14.97\end{array}$ & $\begin{array}{l}2.05 \\
4.44\end{array}$ & $\begin{array}{l}1.20 \\
2.42\end{array}$ & $\begin{array}{l}26 \cdot 19 \\
45 \cdot 31\end{array}$ \\
\hline Total & $\begin{array}{l}\text { Remunoraoibn } \\
\text { Fuerza de trabajo }\end{array}$ & $\begin{array}{l}31.26 \\
29.47\end{array}$ & $\begin{array}{l}19.59 \\
19.27\end{array}$ & $\begin{array}{l}22.17 \\
26.13\end{array}$ & $\begin{array}{l}16.75 \\
17.13\end{array}$ & $\begin{array}{r}10.21 \\
7.98\end{array}$ & $\begin{array}{l}100.00 \\
100.00\end{array}$ \\
\hline
\end{tabular}




\section{Cuadro 2}

DISTRIBUCIÓN DE LA FUERZA DE TRABAJO Y SUS REMUNERACIONES POR GRUPO OCUPACIONAL Y RAMA DE ACTIVIDAD, 1965

Después de los impuestos

(Porcientos)

\begin{tabular}{|c|c|c|c|c|c|c|c|}
\hline \multirow[t]{2}{*}{ Grupo } & & \multicolumn{2}{|r|}{$\mathbf{s}$} & \multirow{2}{*}{$\frac{c t}{I I I}$} & \multicolumn{2}{|l|}{ or } & \multirow[t]{2}{*}{ Total } \\
\hline & & I & II & & IV & v & \\
\hline 1 & $\begin{array}{l}\text { Remuneracion } \\
\text { Fuerza de trabajo }\end{array}$ & $\begin{array}{l}2.02 \\
0.44\end{array}$ & $\begin{array}{l}1.74 \\
0.42\end{array}$ & $\begin{array}{l}2.42 \\
0.58\end{array}$ & $\begin{array}{l}1.80 \\
0.33\end{array}$ & $\begin{array}{l}0.90 \\
0.22\end{array}$ & $\begin{array}{l}8.93 \\
1.99\end{array}$ \\
\hline 2 & $\begin{array}{c}\text { Remuneración } \\
\text { Fuerza de trabajo }\end{array}$ & $\begin{array}{l}6.25 \\
3.18\end{array}$ & $\begin{array}{l}4.42 \\
1.84\end{array}$ & $\begin{array}{l}3.57 \\
2.24\end{array}$ & $\begin{array}{l}3.65 \\
1.92\end{array}$ & $\begin{array}{l}1.47 \\
0.73\end{array}$ & $\begin{array}{r}19.36 \\
9.91\end{array}$ \\
\hline 3 & $\begin{array}{c}\text { Remuneración } \\
\text { Fuerza de trabajo }\end{array}$ & $\begin{array}{l}1.94 \\
1.42\end{array}$ & $\begin{array}{l}1.53 \\
1.28\end{array}$ & $\begin{array}{l}1.66 \\
1.48\end{array}$ & $\begin{array}{l}1.90 \\
0.76\end{array}$ & $\begin{array}{l}0.53 \\
0.42\end{array}$ & $\begin{array}{l}6.56 \\
5 \cdot 36\end{array}$ \\
\hline 4 & $\begin{array}{c}\text { Remuneración } \\
\text { Fuerza de trabajo }\end{array}$ & $\begin{array}{l}12.47 \\
10.15\end{array}$ & $\begin{array}{l}6.68 \\
6.53\end{array}$ & $\begin{array}{l}4.93 \\
6.86\end{array}$ & $\begin{array}{l}8.28 \\
9.68\end{array}$ & $\begin{array}{l}5.85 \\
4.18\end{array}$ & $\begin{array}{l}38.21 \\
37.40\end{array}$ \\
\hline 5 & $\begin{array}{c}\text { Remuneración } \\
\text { Fuerza de trabajo }\end{array}$ & $\begin{array}{r}8.61 \\
14.28\end{array}$ & $\begin{array}{l}5.18 \\
9.20\end{array}$ & $\begin{array}{r}9.78 \\
14.97\end{array}$ & $\begin{array}{l}2.12 \\
4 \cdot 44\end{array}$ & $\begin{array}{l}1.24 \\
2.42\end{array}$ & $\begin{array}{l}26.93 \\
45.31\end{array}$ \\
\hline Total & $\begin{array}{c}\text { Remune ración } \\
\text { Fuerza de trabajo }\end{array}$ & $\begin{array}{l}31.29 \\
29.47\end{array}$ & $\begin{array}{l}19.55 \\
19.27\end{array}$ & $\begin{array}{l}22.41 \\
26.13\end{array}$ & $\begin{array}{l}16.75 \\
17.13\end{array}$ & $\begin{array}{l}9.99 \\
7.98\end{array}$ & $\begin{array}{l}100.00 \\
100.00\end{array}$ \\
\hline
\end{tabular}

decir, que todos los demás grupos perciben salarios superiores al promedio y solamente el último muestra la relación contraria. En el cuadro 4 puede verse el efecto de los impuestos sobre la distribución. Es notorio que la situación del último grupo permanece igual en relación con los cuatro restantes.

Por su parte, la distribución de los pagos en los grupos económicos resulta ser más uniforme; tres grupos tienen salarios medios superiores al nacional, y menores en los dos restantes. El grupo residual parece ser el más beneficiado y el grupo III de industrias metálicas el más bajo. Estas variaciones dependen de la estructura de fuerza de trabajo de las empresas. Nótese que en los grupos de empresas III y IV los ejecutivos medios tienen los menores sueldos en comparación con los otros grupos. Los altos ejecutivos en el grupo I son los mejor pagados.

\section{Cuadro 3}

REMUNERACIONES CON RESPECTO AL SALARIO GLOBAL POR GRUPOS OCUPACIONALES Y RAMAS DE ACTIVIDAD, 1965

Antes de los impuestos

\begin{tabular}{cccccccc}
\hline \multirow{2}{*}{ Grapo } & \multicolumn{5}{c}{ S 0 o t 0 T } & Marginal \\
\cline { 2 - 6 } & I & II & III & IV & V & \\
\hline 1 & 4.86 & 4.38 & 4.52 & 5.91 & 4.32 & 4.77 \\
2 & 1.99 & 2.49 & 1.59 & 1.93 & 2.04 & 1.99 \\
3 & 1.37 & 1.19 & 1.11 & 1.16 & 1.24 & 1.21 \\
4 & 1.23 & 1.09 & 1.70 & 1.84 & 1.44 & 1.02 \\
5 & 0.59 & 0.55 & 0.64 & 0.46 & 0.50 & 0.58 \\
Marginal & 1.06 & 1.02 & 0.85 & 0.98 & 1.28 & \\
\hline
\end{tabular}


En cuanto a los pagos en la escala salarial, salta a la vista la diferencia entre el salario de los altos ejecutivos en relación con los grupos restantes. Asimismo puede verse que los grupos seleccionados sí forman una escala de remuneraciones y que después de los impuestos la situación no cambia mucho.

Cuadro 4

REMUNERACIONES CON RESPECTO AL SALARIO GLOBAL POR GRUPOS OCUPACIONALES Y RAMAS DE ACTIVIDAD, 1965

Después de los impuestos

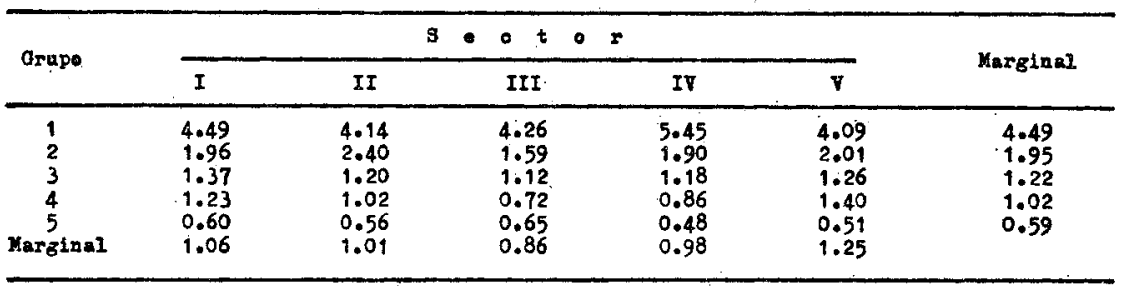

Las diferencias globales que aparecen en los cuadros 5 y 6 antes y después de los impuestos, respectivamente, indican que las diferencias en la escala salarial son mucho mayores que las geográficas, lo cual era de esperarse. El $75 \%$ de las disparidades son de la escala y el $25 \%$ son geográficas. Esto significa que las modificaciones del salario son mucho más importantes cuando la persona asciende en la escala que cuando cambia de empresa dentro del mismo empleo. Las diferencias entre grupos son las mayores en esta categoría, lo cual indica que la mayor probabilidad de modificación del salario ocurre al cambiar de grupo ocupacional, ante las alternativas de permanecer dentro de él o cambiar de empresa.

Si hay alguna asociación sustancial entre el salario y la educación, la experiencia, la responsabilidad y otros factores, el mercado de fuerza de trabajo sí traduce los cambios de estos últimos en una modificación importante del salario, o sea, que la movilidad ocupacional sí trae beneficios importantes al que la practica, en comparación con el cambio de unidad económica al permanecer en el mismo empleo. Sin embargo, esto sucede a saltos y no gradualmente ya que las mayores posibilidades de movilidad ocupacional se presentan en los tres gru-

\section{Cuadro 5}

DifERENCIAS GLOBALES

Antes de los impuestos

\begin{tabular}{|c|c|c|c|c|c|c|}
\hline \multirow{2}{*}{ 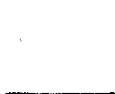 } & \multicolumn{2}{|c|}{ Entre } & \multicolumn{2}{|c|}{ Intra } & \multicolumn{2}{|c|}{ Sume } \\
\hline & absolutag & $\%$ & absolata & $\%$ & absolutas & $\%$ \\
\hline $\begin{array}{c}\text { Empleos } \\
\text { Grupos } \\
\text { Suma }\end{array}$ & $\begin{array}{l}0.1171 \\
0.1601 \\
0.2772\end{array}$ & $\begin{array}{l}31.6 \\
43.1 \\
74.7\end{array}$ & $\begin{array}{l}0.0791 \\
0.0148 \\
0.0939\end{array}$ & $\begin{array}{r}21.3 \\
4.0 \\
25.3\end{array}$ & $\begin{array}{l}0.1962 \\
0.1749 \\
0.3711\end{array}$ & $\begin{array}{r}52.9 \\
47.1 \\
100.0\end{array}$ \\
\hline
\end{tabular}


pos superiores y en poca medida en los dos últimos. Por ejemplo, para que las personas de los grupos "empleados de oficina" y "trabajadores manuales". puedan cambiar a cualquiera de los tres restantes, es necesaria una mejoría en el nivel educativo, principalmente para cambiar al grupo de "personal técnico y profesional". Entre los tres grupos superiores, la movilidad tiene menos fricciones de esta índole. Las posibilidades de cambio en los dos últimos grupos quedan pues limitadas al cambio geográfico o intragrupo.

\section{Cuadro 6}

\section{DIFERENCTAS GLOBALES}

Después de los impuestos

\begin{tabular}{|c|c|c|c|c|c|c|}
\hline & \multicolumn{2}{|c|}{ Bntro } & \multicolumn{2}{|c|}{ Intra } & \multicolumn{2}{|c|}{ Suma } \\
\hline & absolutas & $\%$ & absolutas & $\%$ & Absolutas & $\%$ \\
\hline $\begin{array}{l}\text { Empleos } \\
\text { Grupos } \\
\text { Suma }\end{array}$ & $\begin{array}{l}0.1080 \\
0.1449 \\
0.2529\end{array}$ & $\begin{array}{l}32.2 \\
43.2 \\
75.4\end{array}$ & $\begin{array}{l}0.0695 \\
0.0130 \\
0.0825\end{array}$ & $\begin{array}{r}20.7 \\
3.9 \\
24.6\end{array}$ & $\begin{array}{l}0.1775 \\
0.1579 \\
0.3354\end{array}$ & $\begin{array}{l}52.9 \\
47.1\end{array}$ \\
\hline
\end{tabular}

Al aplicar los impuestos sucede que, aunque las diferencias disminuyen globalmente, su estructura se recarga ligeramente en favor de la categoria entre, esto es, en favor de las diferencias de la escala salarial (ver cuadros 5 y 6). Por la forma de la ley de impuestos, que aplica descuentos fijos a intervalos de salarios, más un descuento proporcional a la diferencia entre el salario y el límite inferior del intervalo, puede deducirse que el gravamen suaviza más las diferencias entre salarios que quedan dentro del mismo intervalo que las de salarios de intervalos distintos. Por esta razón, las diferencias geográficas pierden importancia al aplicar el gravamen. Esto permite hacer consideraciones interesantes sobre la forma de la tarifa, ya que las observaciones anteriores brindan un criterio fundamental para elaborarla. Por ejemplo, podría idearse una con el objetivo de redistribuir más sustancialmente las remuneraciones en la escala salarial, en comparación con las del otro tipo.

Las diferencias entre e intra-empleos son susceptibles de mayor descomposición. En el cuadro 7 aparece una descomposición en el sentido de los grupos ocupacionales para ambas circunstancias, antes y

Cuadro 7

DIFERENCIAS OCUPACIONALES

\begin{tabular}{|c|c|c|c|c|c|c|c|c|}
\hline \multirow[b]{2}{*}{ Orupo de Empleos } & \multicolumn{4}{|c|}{ Antes do 108 Impuestos } & \multicolumn{4}{|c|}{ Despuse do 208 Impueston } \\
\hline & $\begin{array}{l}\text { Fintro em } \\
\text { absolutas }\end{array}$ & ploos & $\begin{array}{l}\text { Intra } \\
\text { absolutas }\end{array}$ & ploog & $\begin{array}{l}\text { linftre } \\
\text { mbsolutas }\end{array}$ & ipleos & $\begin{array}{l}\text { Intra om } \\
\text { absolutas }\end{array}$ & $\begin{array}{r}0 \% \\
0 \%\end{array}$ \\
\hline $\begin{array}{l}\text { Ntos ejecutivos } \\
\text { Ejecuti pos Kedios } \\
\text { Personal Tónioo }\end{array}$ & $\begin{array}{l}0.0033 \\
0.0163\end{array}$ & $\begin{array}{r}2.8 \\
13.9\end{array}$ & $\begin{array}{l}0.0072 \\
0.0170\end{array}$ & $\begin{array}{r}9.1 \\
21.5\end{array}$ & $\begin{array}{l}0.0027 \\
0.0142\end{array}$ & $\begin{array}{r}2.5 \\
13.2\end{array}$ & $\begin{array}{l}0.0060 \\
0.0147\end{array}$ & $\begin{array}{r}8.6 \\
21.1\end{array}$ \\
\hline $\begin{array}{l}\text { profesional } \\
\text { Persona d de Opicina } \\
\text { Trabajadores Manaeles } \\
\text { Suma }\end{array}$ & $\begin{array}{l}0.0026 \\
0.0659 \\
0.0290 \\
0.1171\end{array}$ & $\begin{array}{r}2.2 \\
56.3 \\
24.8 \\
100.0\end{array}$ & $\begin{array}{l}0.0033 \\
0.0448 \\
0.0068 \\
0.0791\end{array}$ & $\begin{array}{r}4.2 \\
56.6 \\
8.6 \\
100.0\end{array}$ & $\begin{array}{l}0.0024 \\
0.0604 \\
0.0282 \\
0.1079\end{array}$ & $\begin{array}{r}2.2 \\
56.0 \\
26.1 \\
100.0\end{array}$ & $\begin{array}{l}0.0031 \\
0.0390 \\
0.0067 \\
0.0695\end{array}$ & $\begin{array}{r}4.4 \\
56.2 \\
9.7 \\
100.0\end{array}$ \\
\hline
\end{tabular}


después de los impuestos. En ellas podemos ver qué grupos ocupacionales tienen las mayores disparidades, dentro de la clasificación establecida. Por una parte, se observa que las fluctuaciones de los salarios en ambas categorías entre e intra tienen una distribución muy similar. Los grupos "personal de oficina" (el más dispar) y "trabajadores manuales", juntos, dan cuenta de la mayor parte de las diferencias: $81.1 \%$ en el caso entre, y $65.2 \%$ en el caso intra. Tal vez esta concentración de las diferencias se deba a una agrupación inadecuada de los empleos; sin embargo, la importancia relativa de las diferencias intra en el grupo "empleados de oficina" sugiere que, ante una situación educativa más o menos uniforme, los otros determinantes del salario juegan un papel importante dentro de estos grupos. Parece ser que en estos dos grupos es donde la diferencia entre salarios iniciales y los más frecuentes es más acentuada, además de que su peso relativo es el mayor. Lo anterior no parece muy compatible con el hecho de que los empleos más frecuentes y bien tipificados tienen salarios más definidos, debido a las influencias sindicales y a la regularidad de las funciones, en especial en el caso de empleados de oficina.

Por su parte, las diferencias en los tres primeros grupos, entre las cuales las de ejecutivos medios son las más importantes, sólo explican menos de la quinta parte del total en el caso entre y casi una tercera parte en el caso intra. Nótese que las contribuciones intra son mayores, en particular en el segundo grupo ocupacional, lo cual sugiere que los factores de escasez de empleados calificados realzan las disparidades geográficas. Es notable la baja contribución del grupo técnico y profesional, compuesto por ocupaciones de índole técnica más que administrativa. Los impuestos acentúan las diferencias (últimas dos columnas del cuadro 7) del último grupo en ambas categorías, así como las intra del grupo de personal técnico y profesional. Esto es resultado de que la tarifa impositiva tiene una gradación menos acentuada en los primeros intervalos.

\section{REFERENCIAS BIBLIOGRAFICAS}

[1] P. Uribe, "Concentración demográfica y estructura urbana: un enfoque vía teoría de la información", Demograffa Y ECONOMf́a, Vol. I, Núm. 2, 1967, pp. 151-180.

[2] John T. Dunlop, "The Task of Contemporary Wage Theory", en New Concepts in Wage Determination, Nueva York, McGraw Hill, 1957.

[3] J. R. Hicks, The Theory of Wages, Macmillan, 1932.

[4] Instituto de Participación de las Utilidades en el Salario (IPAUS), Confederación Patronal de la República Mexicana (COPARMEX), Encuesta técnica sobre salarios y prestaciones, México, Area metropolitana, 1966.

[5] R. Perlman, compilador, Wage Determination - Market or Power Forces?, Boston, D. C. Heath, 1964. Ver en particular la discusión de la parte 1 de Richard A. Lester.

[6] F. F. Reza, Introduction to Information Theory, Nueva York, McGraw Hill, 1961.

[7] C. R. Rao, Linear Statistical Inference and Its Applications, Nueva York, John Wiley, 1967, p. 47. 